\title{
A 39-year-old female immigrant with chronic diarrhea
}

\author{
Omar N Nadhem MD ${ }^{1}$, Ruba A Halloush MD $^{2}$, Faisal A Khasawneh MD ${ }^{3}$
}

\begin{abstract}
A 39-year-old female immigrant from Yemen presented with several months' history of diarrhea. The stool was loose in consistency and brown in colour. There was no blood or mucus noted. She reported a dull periumbilical abdominal pain, poor appetite and weight loss but had not experienced any fever, nausea or vomiting. The patient immigrated to the United States after she was married, 18 years previously. She reported a negative tuberculin skin test at the time of intake.

On physical examination, she was cachectic but afebrile, with a normal heart rate and blood pressure. Chest auscultation revealed clear lung fields and regular heart sounds. The abdomen was soft, not tender and without organomegaly. She had no skin rash and no lymphadenopathy.

Laboratory test results revealed a hemoglobin level of $85 \mathrm{~g} / \mathrm{L}$ and a white blood cell count of $9.4 \times 10^{9}$ cells/L without eosinophilia. Her blood chemistry, including kidney and liver function tests, were within normal limits. Stool microscopy for ova and parasites and stool culture were unrevealing. An abdominal computed tomography scan showed segmental thickening in the right colon (Figure 1A). Colonoscopy was performed and tissue biopsies were obtained. Hematoxylin and eosinstained slides are shown in Figures 1B and 1C.

What is your diagnosis?
\end{abstract}

\section{DIAGNOSIS}

The colon biopsy showed granulomatous inflammation composed of epithelioid histiocytes and multinucleated giant cells, and acid-fast bacilli staining showed mycobacteria (Figures $1 \mathrm{~B}$ and 1C). She was diagnosed with segmental tuberculosis (TB) of the colon. She had no evidence of lung disease. The patient was started empirically on rifampin (RIF), isoniazid (INH), ethambutol and pyrazinamide. The tissue culture grew Mycobacterium tuberculosis that was susceptible to all of the above anti-TB medications. She finished a seven-month course without significant side effects. She was symptom free at oneyear follow-up.

\section{DISCUSSION}

TB causes significant morbidity and mortality worldwide (1). Immigration and the HIV epidemic led to resurgence of this infection (1). Poverty, malnutrition, overcrowding and HIV coinfection facilitates the spread of this ailment (2). The incidence of TB has been declining in the United States and other Western countries (1,3). In 2012, the incidence of TB in the United States was 3.2 per 100,000 population, a significantly lower number compared with its incidence in 1993, which was 9.7 per 100,000 population. Despite this decline, the number of extrapulmonary $\mathrm{TB}$ cases have been increasing; the expanding immigrant populations, HIV and improved diagnostic tools partially explain this $(3,4)$.

Extrapulmonary TB accounts for $20 \%$ of diagnosed TB cases in immunocompetent individuals and up to $50 \%$ of TB cases in immunocompromised hosts (5). Gastrointestinal (GI) TB is the sixth most frequent form of extrapulmonary TB, after lymphatic, genitourinary, bone and joint, miliary and meningeal TB (6). It can affect any part of the GI tract, with ileocecal involvement being the most common. GI TB infection is acquired following one of four mechanisms: swallowing infected sputum, hematogenous spread from active pulmonary or miliary $\mathrm{TB}$, ingestion of contaminated milk or food, and contiguous spread from adjacent organs (7). Only $15 \%$ of patients with GI TB show evidence of pulmonary disease (7).

Isolated segmental TB of the colon is rare and accounts for $<10 \%$ of GI TB (7). It is subacute in presentation, with symptoms developing over weeks to months. Patients often complain of nonspecific abdominal pain (the most common symptom, occurring in $80 \%$ to $90 \%$ of cases), fever, weight loss, night sweats and diarrhea (8). Physical examination may reveal cachexia, right lower abdominal mass - in cases of ileocecal involvement - and ascites.

Routine blood tests may reveal chronic anemia and high erythrocyte sedimentation rate; however, the white blood cell count is usually normal. Tuberculin skin test (TST) and interferon-gamma release assay (IGRA) may be positive, but neither can differentiate between active and latent TB infection. In meta-analyses, TST sensitivity and specificity were reported to be $71 \%$ and $97 \%$, respectively (9). In two small studies investigating intestinal TB, reported TST sensitivity was $64 \%$ and $86 \%(10,11)$. The reported sensitivity of IGRA in the diagnosis of extrapulmonary TB is between $71 \%$ and $93 \%$ and the specificity ranged between $56 \%$ and $89 \%$, based on the prevalence of TB in the study population $(12,13)$. IGRA has not specifically been studied in intestinal TB, but was useful in the diagnosis described in two case reports (14). In intestinal TB, stool studies are nonspecific (2).

Contrast-enhanced computed tomography of the abdomen and pelvis is the imaging modality of choice in diagnosing colonic TB (15). Imaging may reveal one or more of the following: enlarged lymph nodes, asymmetric bowel wall thickening or an inflammatory mass, strictures, ascites and, rarely, pancolitis.

The definitive diagnosis of colonic TB hinges on the combination of consistent histology and positive mycobacterial culture of biopsy material obtained by colonoscopy. Colonoscopic findings of colonic TB show one of three forms: ulcerative, hypertrophic or ulcerohypertrophic, or fibrinous (7). For optimal diagnostic yield, at least four to six deep endoscopic biopsies should be obtained from the ulcer base and its margins (16). Of note, acid-fast bacilli are identified in a minority $(<33 \%)$ of biopsy specimens (17). Culture of biopsy material remains the gold standard but is slow. Polymerase chain reaction testing of biopsy specimens can facilitate the diagnosis and results can be reported within $48 \mathrm{~h}$; however, this is not widely available $(9,18)$. The role of Xpert MTB/RIF (Cepheid, USA), a polymerase chain reaction assay, in the diagnosis of extrapulmonary $\mathrm{TB}$ was evaluated in 1476 extrapulmonary specimens (19). The assay showed an overall sensitivity and specificity of $81.3 \%$ and $99.8 \%$, respectively. For biopsies, urine, pus and cerebrospinal fluid, the sensitivity exceeded $85 \%$; meanwhile, it was slightly $<80 \%$ for gastric aspirates and $<50 \%$ for ascites and other cavitary fluid samples.

\footnotetext{
${ }^{1}$ Department of Internal Medicine, Texas Tech University Health Sciences Center; ${ }^{2}$ Amarillo Pathology Group; ${ }^{3}$ Section of Infectious Diseases,

Department of Internal Medicine, Texas Tech University Health Sciences Center, Amarillo, Texas, USA

Correspondence: Dr Faisal A Khasawneh, Section of Infectious Diseases, Department of Internal of Medicine, Texas Tech University Health

Sciences Center, 1400 South Coulter Street, Amarillo, Texas 79106, USA. Telephone 806-354-5480, fax 806-354-5765,

e-mail faisal.khasawneh@ttuhsc.edu
}

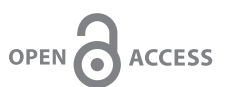

This open-access article is distributed under the terms of the Creative Commons Attribution Non-Commercial License (CC BY-NC) (http:/ creativecommons.org/licenses/by-nc/4.0/), which permits reuse, distribution and reproduction of the article, provided that the original work is properly cited and the reuse is restricted to noncommercial purposes. For commercial reuse, contact support@pulsus.com 


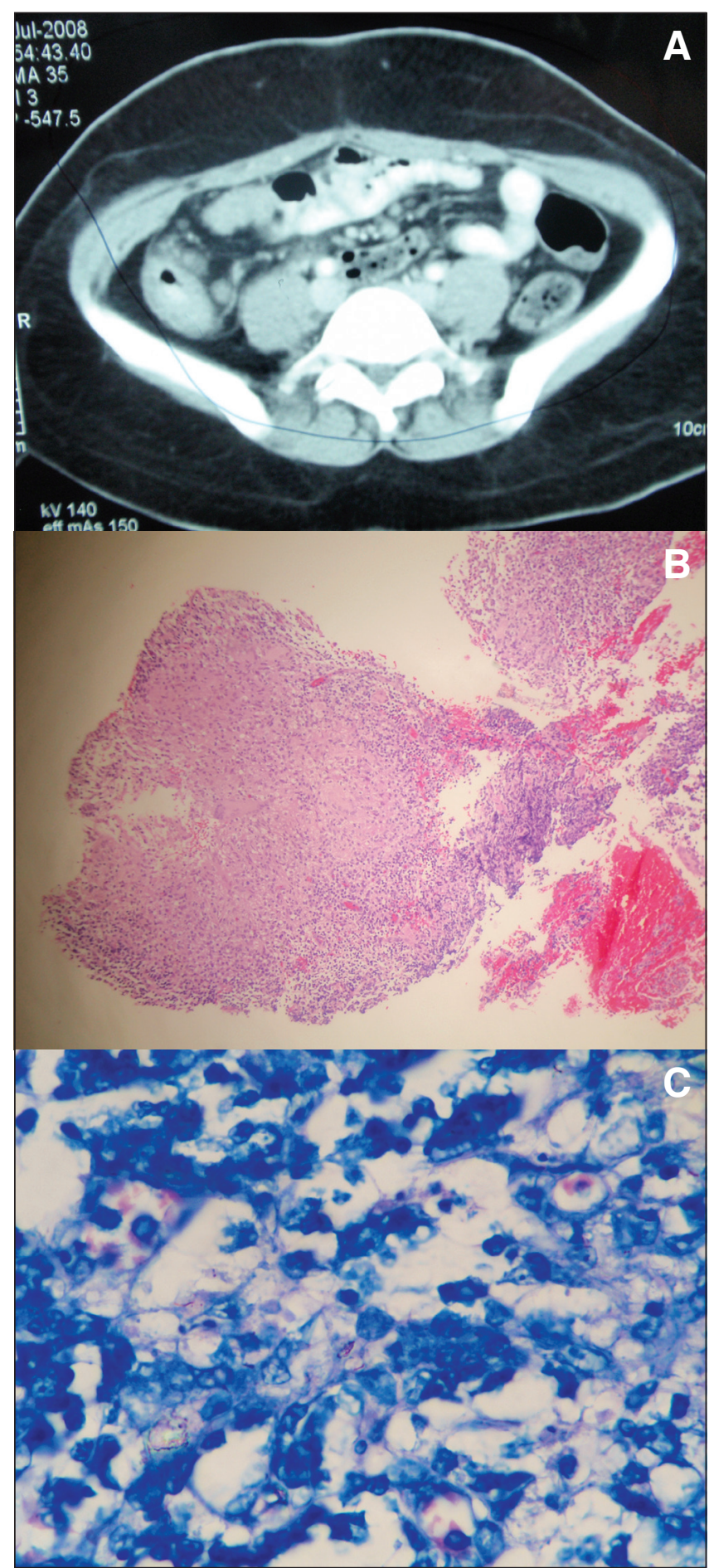

Figure 1) A Computed tomography section of the abdomen with contrast showing thickened right colonic wall. B Hematoxylin and eosin-stained section of the colon biopsy showing granulomatous inflammation composed of epithelioid histiocytes and multinucleated giant cells. Original magnification $\times 100$. C Acid-fast bacillus stain showing mycobacteria. Original magnification $\times 400$

Despite the above, the diagnosis of TB colitis can remain uncertain (20). For situations in which there is high index of suspicion of TB on clinical grounds, anti-TB therapy is appropriate.

The differential diagnoses of colonic TB include inflammatory bowel disease, carcinoma and amebiasis (2). Ileocecal TB has broader differential diagnoses including lymphoma, appendicular abscess, enteric fever, actinomycosis and yersiniosis (2).
TB colitis treatment consists of RIF, INH, pyrazinamide and ethambutol for two months, followed by four to seven months of RIF and INH (1). Treatment is commonly started empirically based on high clinical suspicion or suggestive preliminary test results. In Western countries and mainly among immigrant populations, multidrug-resistant TB (MDR-TB) and extensive drug-resistant TB are on the rise, making reliable empirical therapeutic recommendations difficult (21). A recent study evaluated the prevalence of MDR-TB among foreign-born persons in Alberta (22). Between 2002 and 2011, MDR-TB prevalence was $2.1 \%$, a sharp increase from the preceding decade, during which the prevalence was $0.56 \%$. Resistant TB strains should be suspected in immigrants from high-risk areas and empirical therapy should, preferably, be based on genotypic drug susceptibility testing. Delaying therapy in stable patients of this group, if they can be quarantined so as not to infect others, to allow for susceptibility-guided therapy as opposed to empirical therapy that may be inadequate (risking failure and the further development of resistance) is a consideration.

Bowel obstruction is the most common complication and may be due to a progressive stricture or adhesions (23). Other potential complications include perforation with secondary peritonitis, gastrointestinal bleed and fistula formation $(24,25)$. TB colitis patients demonstrate clinical improvement within two weeks of therapy commencement. Surgical treatment is reserved for bowel obstruction, bowel perforation and massive bleeding (26).

\section{REFERENCES}

1. Zumla A, Raviglione M, Hafner R, von Reyn CF. Tuberculosis.

N Engl J Med 2013;368:745-55.

2. Donoghue HD, Holton J. Intestinal tuberculosis. Curr Opin Infect Dis 2009;22:490-6.

3. Kruijshaar ME, Abubakar I. Increase in extrapulmonary tuberculosis in England and Wales 1999-2006. Thorax 2009;64:1090-5.

4. Sandgren A, Hollo V, van der Werf MJ. Extrapulmonary tuberculosis in the European Union and European Economic Area, 2002 to 2011. Euro Surveill 2013;18:20431.

5. Farer LS, Lowell AM, Meador MP. Extrapulmonary tuberculosis in the United States. Am J Epidemiol 1979;109:205-17.

6. Dasgupta A, Singh N, Bhatia A. Abdominal tuberculosis: A histopathological study with special reference to intestinal perforation and mesenteric vasculopathy. J Lab Physicians 2009;1:56-61.

7. Marshall JB. Tuberculosis of the gastrointestinal tract and peritoneum. Am J Gastroenterol 1993;88:989-99.

8. Alvares JF, Devarbhavi H, Makhija P, Rao S, Kottoor R. Clinical, colonoscopic, and histological profile of colonic tuberculosis in a tertiary hospital. Endoscopy 2005;37:351-6.

9. Pai M, Minion J, Steingart K, Ramsay A. New and improved tuberculosis diagnostics: Evidence, policy, practice, and impact. Curr Opin Pulm Med 2010;16:271-84.

10. Sato S, Yao K, Yao T, et al. Colonoscopy in the diagnosis of intestinal tuberculosis in asymptomatic patients. Gastrointest Endosc 2004;59:362-8.

11. Bhargava DK, Kushwaha AK, Dasarathy S, Shriniwas, Chopra P. Endoscopic diagnosis of segmental colonic tuberculosis. Gastrointest Endosc 1992;38:571-4.

12. Feng Y, Diao N, Shao L, et al. Interferon-gamma release assay performance in pulmonary and extrapulmonary tuberculosis. PloS One 2012;7:e32652.

13. Lavender TW, Barrett A, Magee J, Ong EL. Interferon-gamma release assays in the diagnosis of active tuberculosis disease in a low-incident setting: A 5-year review of data. Clin Microbiol Infect 2013;19:1078-81.

14. Caputo D, Alloni R, Garberini A, et al. Experience with two cases of intestinal tuberculosis: Utility of the QuantiFERON-TB Gold test for diagnosis. Surg Infect 2008;9:407-10.

15. Pereira JM, Madureira AJ, Vieira A, Ramos I. Abdominal tuberculosis: Imaging features. Eur J Radiol 2005;55:173-80.

16. Pulimood AB, Peter S, Ramakrishna B, et al. Segmental colonoscopic biopsies in the differentiation of ileocolic tuberculosis from Crohn's disease. J Gastroenterol Hepatol 2005;20:688-96.

17. Balamurugan R, Venkataraman S, John KR, Ramakrishna BS. PCR amplification of the IS6110 insertion element of Mycobacterium tuberculosis in fecal samples from patients with intestinal tuberculosis. J Clin Microbiol 2006;44:1884-6. 
18. Wiener RS, Della-Latta P, Schluger NW. Effect of nucleic acid amplification for Mycobacterium tuberculosis on clinical decision making in suspected extrapulmonary tuberculosis. Chest 2005;128:102-7.

19. Tortoli E, Russo C, Piersimoni C, et al. Clinical validation of Xpert MTB/RIF for the diagnosis of extrapulmonary tuberculosis. Eur Respir J 2012;40:442-7.

20. Giouleme O, Paschos P, Katsaros M, et al. Intestinal tuberculosis: A diagnostic challenge - case report and review of the literature. Eur J Gastroenterol Hepatol 2011;23:1074-7.

21. Minion J, Gallant V, Wolfe J, Jamieson F, Long R. Multidrug and extensively drug-resistant tuberculosis in Canada 1997-2008: Demographic and disease characteristics. PloS One 2013;8:e53466.

22. Long R, Langlois-Klassen D. Increase in multidrug-resistant tuberculosis (MDR-TB) in Alberta among foreign-born persons:
Implications for tuberculosis management. Can J Public Health 2013;104:e22-7.

23. Nagi B, Kochhar R, Bhasin DK, Singh K. Colorectal tuberculosis. Eur Radiol 2003;13:1907-12.

24. Alvi AR, Tanveer Ul H, Pardhan A. Abdominal tuberculosis with massive jejunal haemorrhage. J Coll Physicians Surg Pak 2013;23:509-11.

25. Gani R, Andronikou S. Enterovesical fistula in an HIV patient reactivation of tuberculosis as part of IRIS. S Afr J Surg 2012;50:100-1.

26. Tan KK, Chen K, Sim R. The spectrum of abdominal tuberculosis in a developed country: A single institution's experience over 7 years. J Gastrointest Surg 2009;13:142-7. 


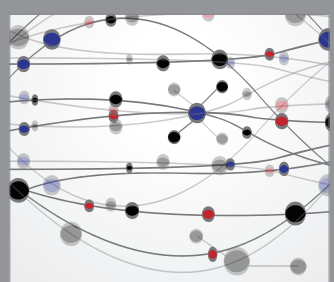

The Scientific World Journal
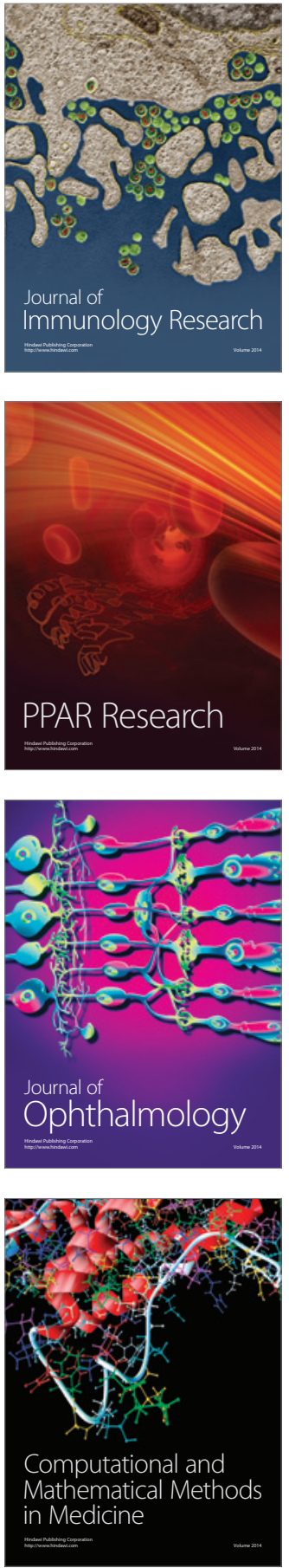

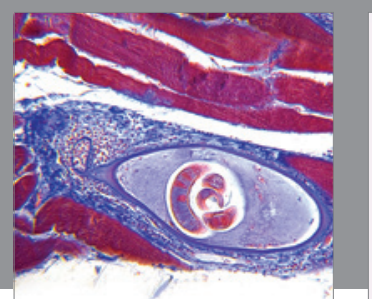

Gastroenterology Research and Practice

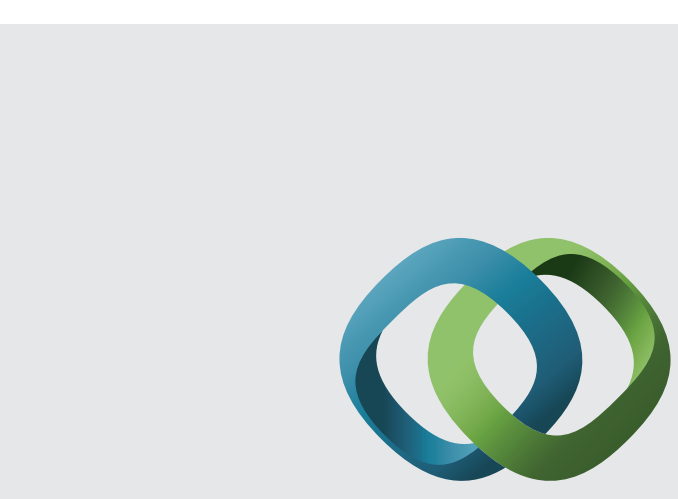

\section{Hindawi}

Submit your manuscripts at

http://www.hindawi.com
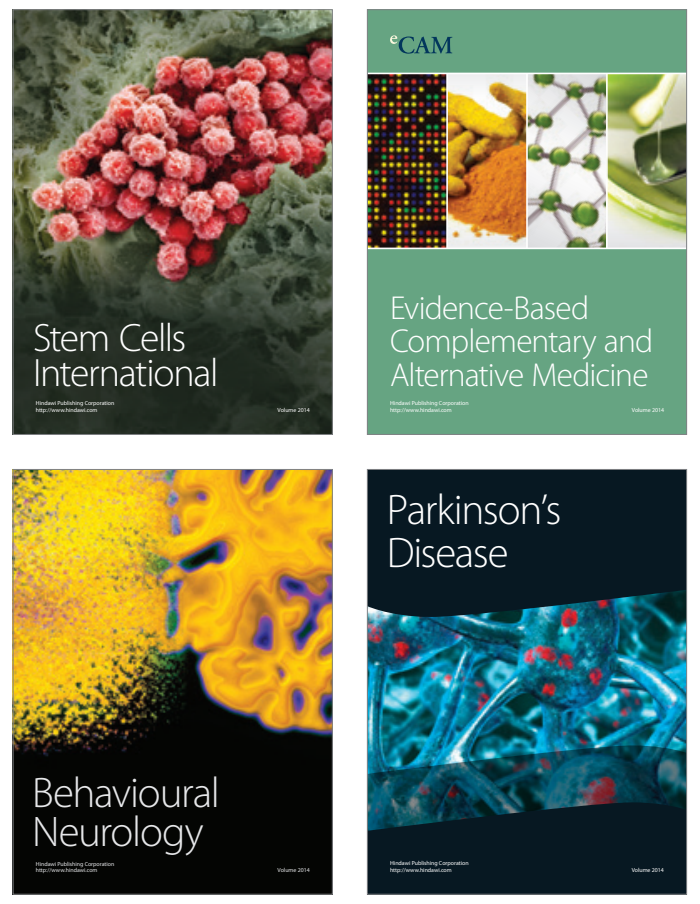
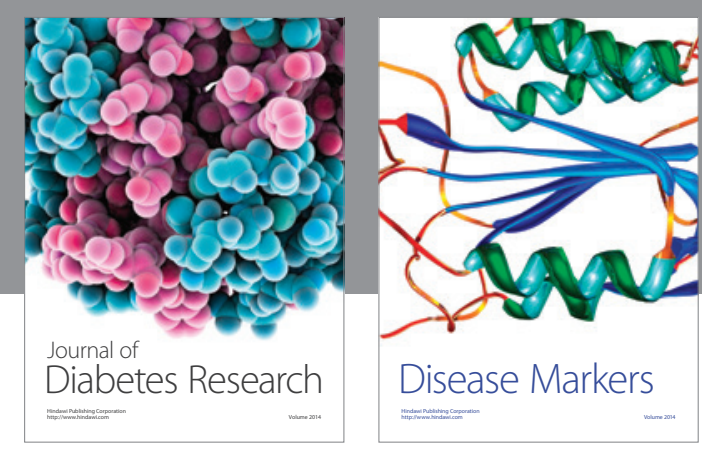

Disease Markers
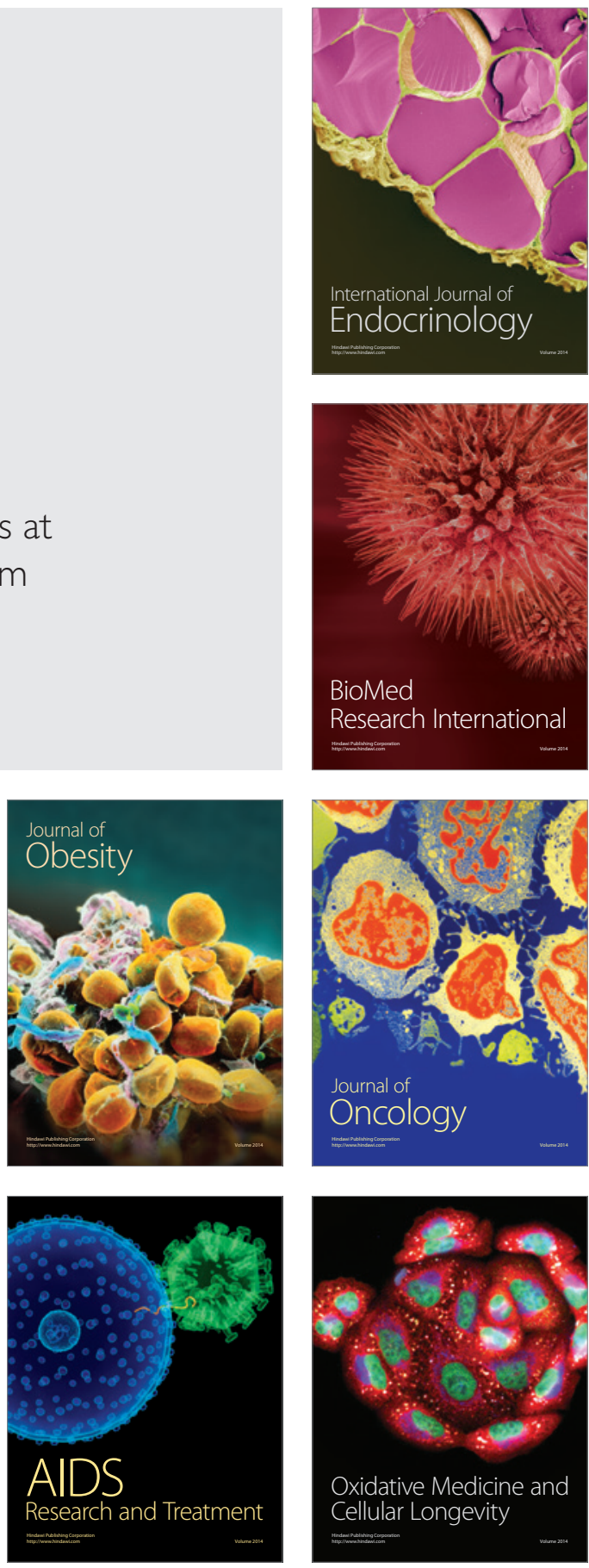